\title{
The Role of Imaging in Diagnosis of Urolithiasis and Nephrolithiasis-A Literature Review Article
}

\author{
Abhinandan Gupta*, Sheng Li, Guanghai Ji, Hao Xiong, Jie Peng, Jingbai Huang \\ Department of Medical Imaging and Nuclear Medicine, The First Affiliated Peoples Hospital of Jing Zhou, Yangtze University, \\ Jingzhou, China \\ Email: *abhinandan7400@gmail.com
}

How to cite this paper: Gupta, A., Li, S., Ji, G.H., Xiong, H., Peng, J. and Huang, J.B. (2019) The Role of Imaging in Diagnosis of Urolithiasis and Nephrolithiasis-A Literature Review Article. Yangtze Medicine, 3, 301-312.

https://doi.org/10.4236/ym.2019.34029

Received: April 6, 2019

Accepted: September 14, 2019

Published: September 17, 2019

Copyright () 2019 by author(s) and Scientific Research Publishing Inc. This work is licensed under the Creative Commons Attribution International License (CC BY 4.0).

http://creativecommons.org/licenses/by/4.0/

\begin{abstract}
Urolithiasis or nephrolithiasis is a common ailment in the emergency room. The clinical presentation of a kidney stone includes fever, nausea, vomiting, acute flank pain radiating to the groin or the back. The pain is often described as stabbing and there is tachycardia, with or without hematuria for the severe patient. For the triad for urinary or kidney stones, some people say they are fever, vomiting, and acute flank pain. So in acute setting analgesia is given with or without an antiemetic to prevent vomiting IV fluids administered carefully. Noncontrast computed tomography (CT) is the gold standard for diagnosis. Most of urinary stones get washouts spontaneously if it is less than $5 \mathrm{~mm}$ without any intervention. However, if intervention is required either it is done by elective or as soon as possible by the intervention. I mean surgical management and surgical management will depend on how big the kidney stone is in there as well as where the kidney stone is if it is within the ureter or within the actual kidney.
\end{abstract}

\section{Keywords}

Urolithiasis, Nephrolithiasis, Kidney Stone, Staghorn Calculus, Ultrasound, X-Ray, CT, MRI, Shockwave Lithotripsy

\section{Introduction}

Kidney stone or Urinary tract stones have many names including nephrolithiasis/urolithiasis [1] which is actually formation of stones along the nephrolithiasis or urinary tract which is stones forming specifically in the nephron or the kidneys to make things even more confusing; kidney stones or urinary can also be 
called renal or urinary calculi. Calculi essentially mean stones [2]. However these words are used interchangeably but essentially mean the same thing of kidney stones. In order to understand kidney stone, we have to revise the anatomy of the adrenal glands which are endocrine glands sitting above the kidneys. The kidneys form urine, urine will then travel down the ureter and be stored in the bladder. The bladder cans stretch and once full we urinate; the urine will flow through the urethra and then out. The ureter has three sites of constriction where it contracts smooth muscle and these are important to know because these are the sites where narrowing can occur and also the site where urinary stones can lodge the sites of ureter constriction including the pelvic ureteric junction [3], the pelvic brim and the vesicoureteric junction [4]. Vesicoureteric junction is actually the connection between the ureter and the urinary bladder which is sort behind and beneath the urinary bladder. Kidney is organ responsible for filtering the blood and disposing of waste; it's important for regulating blood pressure and regulating electrolyte balance. It is also responsible for producing some important hormones such as erythropoietin and activation of vitamin $\mathrm{D}$ [5]. In the adrenal glands which are again the endocrine glands that sit above our kidneys, there is the ureter looking inside the kidney consisting of pyramids called the medulla pyramids. Surrounding the medulla pyramids is the cortex. The medulla pyramids join the tip of the Medulla pyramids, join and form calyx, and the calyxes then join together and form and drain into the renal pelvis. The renal pelvis has a renal artery and renal vein entering and exiting it residing in the region of and within the medulla pyramids are the functional units of the kidneys called nephrons. Nephrons are structures which filter our blood they secrete wastes and allow reabsorption of things into the bloodstream. Thus it has a main role in regulating electrolyte and fluid balance in our body. The head of the nephron is the Bowman's capsule where the afferent arteriole brings blood in forming the glomerulus and then we have the efferent arteriole leaving the glomerulus. Once filtering has occurred within the Bowman's capsule the filtrate will travel along the tubules of the nephron. Firstly it will pass the proximal convoluted tubules then it will go down towards Loop of Henle, the distal convoluted tubules and then finally the collecting duct. Looking more closely at the tubules cells that line the nephron tubules are predominantly cuboidal epithelial cells within tubules of the nephron crystal like structures fall (as we can see in Figure 1). The crystal like structures is essentially precipitance of some electrolytes that have accumulated there. This crystal is actually a urinary stone if the crystal is small it will just pass in the urine but if it remains in the kidneys in the nephron it can grow bigger and become a kidney stone. A kidney stone again is basically a big crystal.

So, in most of the patient Renal colic, hematuria [6] [7] and a positive at plain film X-ray is a typical presentation for $90 \%$ of people who show up with Urolithiasis some people can have vague abdominal pain that it's kind of like some other abdominal inflammation for example diverticulitis or appendicitis and then some people will have real acute abdominal pain and flank pain, nausea, 


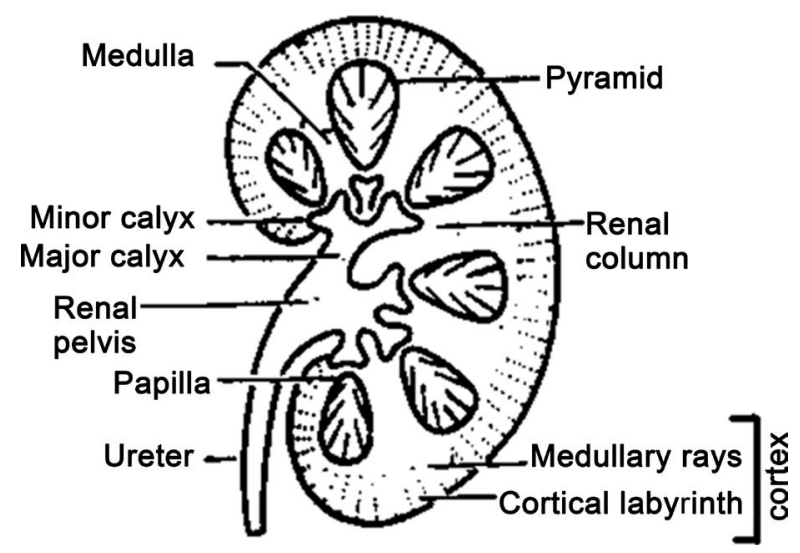

Figure 1. Structure of kidney.

vomiting can be seen urinary urgency and frequency and penile and testicular pain are also symptoms that can show up in a presentation.

\section{Discussion}

The kidney stone can lead to an obstruction the obstruction within the tubules can create a buildup of pressure in the tubules. This pressure can cause irritation and this irritation is read by the brain as renal colic. There is also an inflammatory process going on due to the obstruction and this also leads to the renal colic the pain we feel when there is urinary stone in the body. Alternatively the urinary stone can large or get stuck within the ureter. remember the sites of constriction of the ureter, the stones can get stuck there and when this happens irritation and pain can occur due to stretching of the fibers that are there that which are caused again by the increased in pressure within the ureter with this increase in pressure proximally to the site of obstruction and with the irritation going on edema can occur and the ureter will contract more vigorously trying to push the stone out this is called hyperperistalsis.

So with this in mind the clinical presentation of kidney stones can include acute flank pain which can radiate to the back or towards the groin and the flanks there can be associated fever because of the inflammation and nausea and vomiting there can be also urinary frequency and urgency, hematuria, the person may present to be obese (as we can see in Table 1).

The risk factors for developing a kidney stone a High protein diet [8] [9], high salt diet, male, Caucasian, obesity, dehydration, medications including antacids, carbonic anhydrase inhibitors, other drugs like indinavir, acyclovir, sulfadiazine, triamterene taking four different types of infections. These can cause your intake to crystallize and create stones. sodium and calcium containing medications also increases the risk of developing kidney stones crystal urea is also risk factor as well as having a family history [10] [11]. These risk factors will lead to a number of things first some of these risk factors will increase urinary solute concentration including concentration of calcium, uric acid, and calcium oxalate and sodium [12]. Hypertension, obesity [13] and gout all can lead to renal stones. 
Table 1. Urolithiasis or nephrolithiasis.

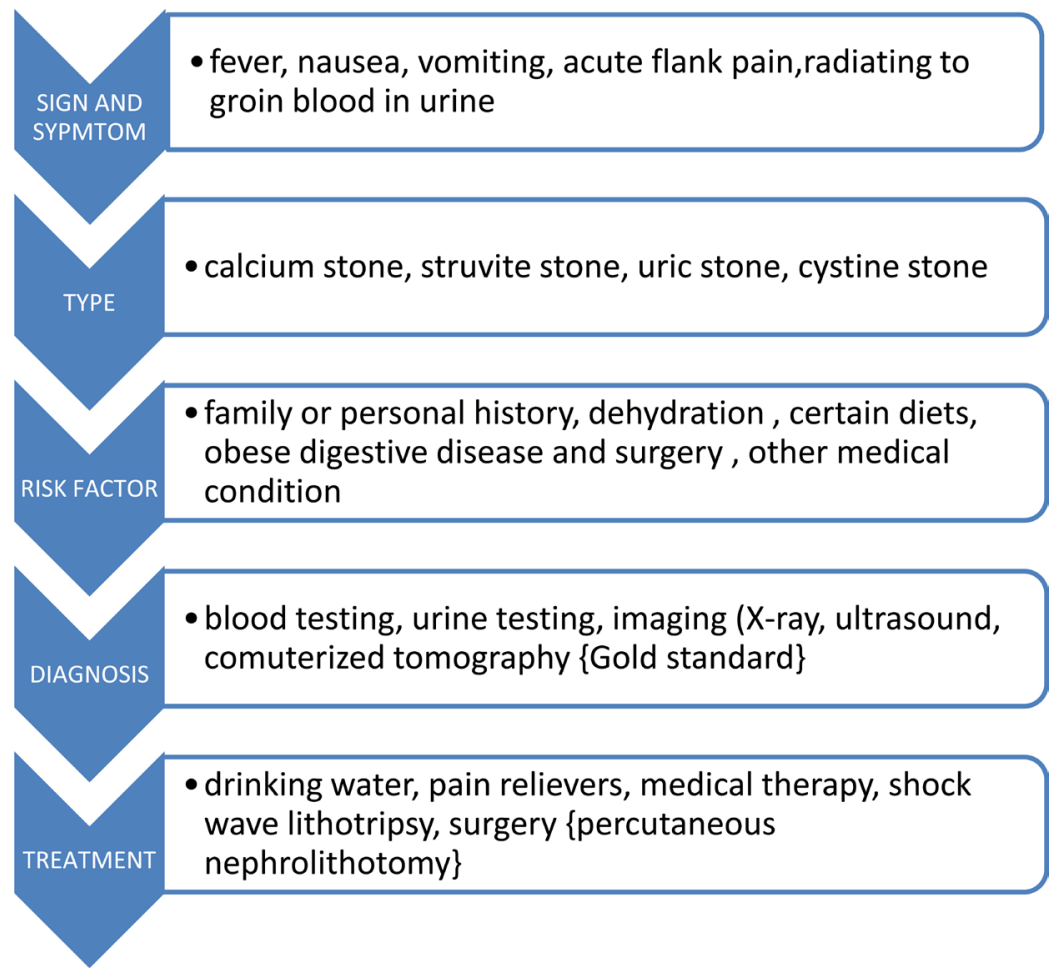

hypertension can cause an increase in calcium, a secretion gout increased uric acid secretion low fluid and fluid intake just makes a higher concentration of the urine and makes the urine more likely to crystallize acidic urine can make acidic substances crystallize and UTI with infection from urease positive bugs like Proteus [14] and Klebsiella can lead to pneumonia formation which can lead to the formation of history white stones.

Some of these risk factors will also decrease the stone forming inhibitors which includes citrate and magnesium the increase in urinary solute and the decrease in urinary stone inhibitors cause urine supersaturation leading to urinary crystal formation or urinary stone formation a decrease in urinary volume such as in dehydration and an excessive increase or decrease in urinary $\mathrm{pH}$ also contributes to urine supersaturation.

Urolithiasis or nephrolithiasis can be different kinds of stones from different sources different underlying causes but most of them are made of calcium or partially made of calcium which means that most of them can also be seen on $\mathrm{x}$-ray because calcium-based stones and are a radiopaque some of these others are as well like struvite stones like you see in this figure here is a staghorn calculus [15] that is a struvite stone most likely from some kind of a UTI cysteine stones can also be radiopaque [15] but uric acid stones are not (as we can see in Figure 2).

DIFFENTIAL DIAGNOSIS: There are other things that percent it similarly to nephrolithiasis including renal cell carcinoma which can also cause blockage or obstruction of the renal system or the ureters, Ectopic pregnancy can have a 


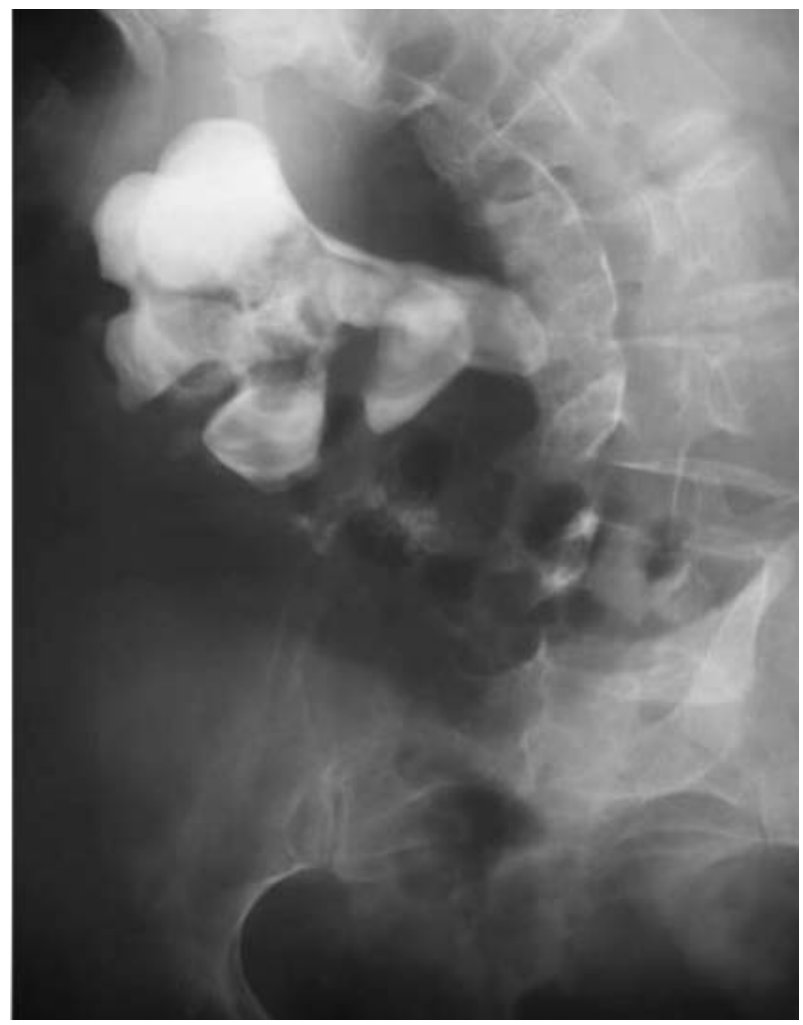

Figure 2. X-ray of staghorn calculus.

similar pain, Aortic aneurysm rarely can have a similar presentation as well as appendicitis.

INVESTIGATION: Suspected renal stones include a full blood count, CRP magnesium calcium phosphate levels(MCP) urinalysis which may show hematuria, a 24-hour urine [5] [16] calcium level, phosphate level, oxalate, urate, cystine, xanthine levels and this can show us what type of kidney stone he might be $\mathrm{X}$-ray can be performed to detect a kidney stone.

On ultrasound finding kidney has 3 basic portions. Outermost is renal capsule followed by renal parenchyma and finally central Sinus. The renal parenchyma consists of outer echogenic cortex \& inner hypoechoic medulla. Medulla consists of hypoechoic triangular pyramids separated by columns of Bertini which arise as invaginations from cortex. In majority of the normal cases, corticomedullary differentiation can be made especially when the patient is adequately hydrated so that the prominent pyramids stand out against the background of renal cortex. Thickness of cortex and medulla are almost same, thus corticomedullary width ratio is nearly $1: 1$. Centre of the kidney has collecting system called renal sinus \& this area is markedly hyperechoic due to renal sinus fat and fibrous tissue. [17] These hyperechoic areas are separated by sonolucent areas in cases of hydronephrosis, peripelvic cysts or crossing renal vessels (as we can see in Figure 3).

Renal calculus-Stone within the kidney usually causes dull pain in the loin. Stone within the renal calyx less commonly produces apparent dilatation of calyx. However when within the ureter, generalized pelvicalyceal dilatation is 
usually seen. Ureteric calculus, unlike the renal calculus usually produces severe colicky pain.

On Ultrasound calculus is diagnosed when hyperechoic area (due to calcification) is seen with acoustic shadowing.

Ultrasound cannot differentiate calcification from other causes (e.g. calcified haematoma) from that of calculus.

However acalcified opacity within the renal sinus is usually of calculus.

An ultrasound can also detect a kidney stone an ultrasound may show an acoustic shadowing, ultrasound may also reveal hydronephrosis [18] [7] (as we can see in Figures 4-7) if the obstruction is within the ureter causing backflow of urine which will dilate the ureter. [19] (as we can see in Figure 8).

The gold standard is for now helical CT for imaging as we noted earlier plain films can most often pick up radiopaque stones but they will not pick up for

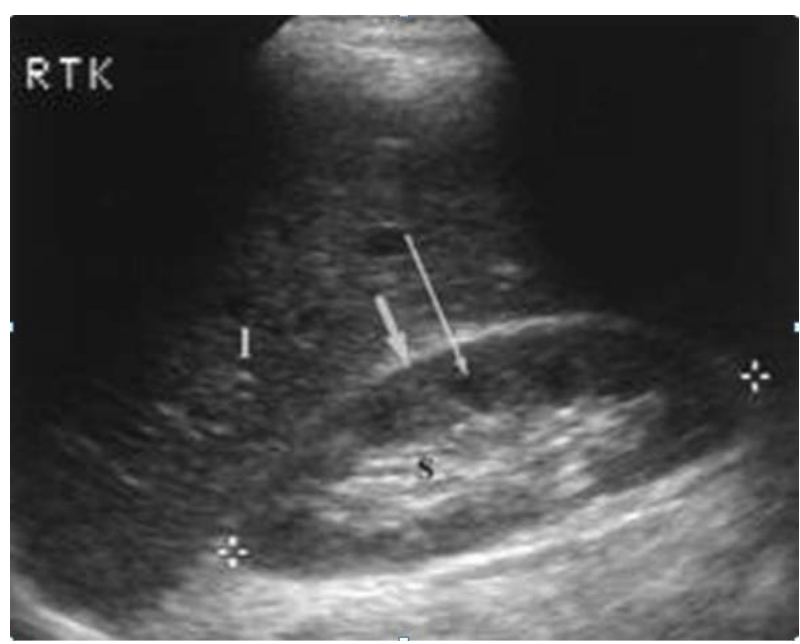

Figure 3. Scan showing hypo echoic pyramids within the medulla(long arrow). Pyramids are separated by columns of Bertini, which are inward protrusions of the cortex into the medulla. (Short arrow-Renal capsule).

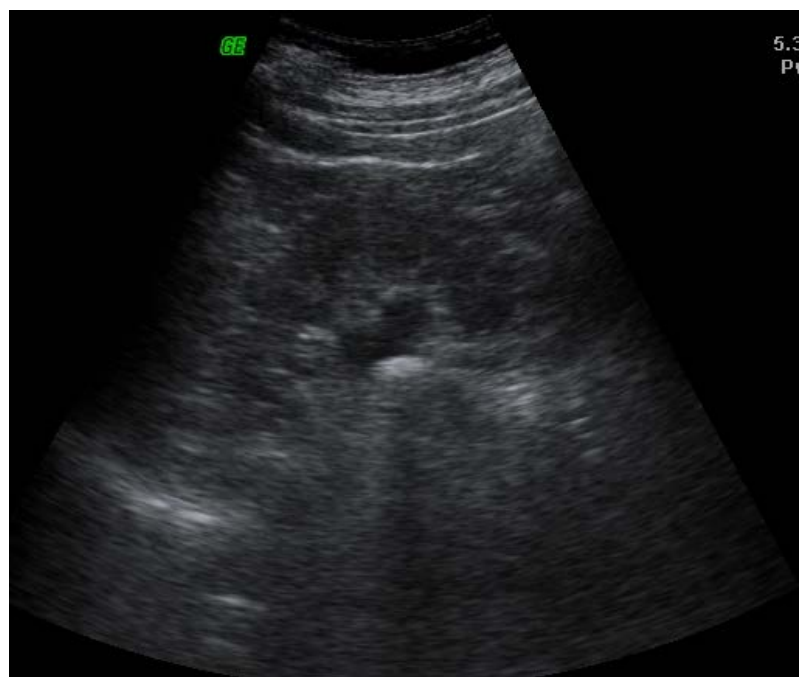

Figure 4. Renal pelvic calculus with mild hydronephrosis. 


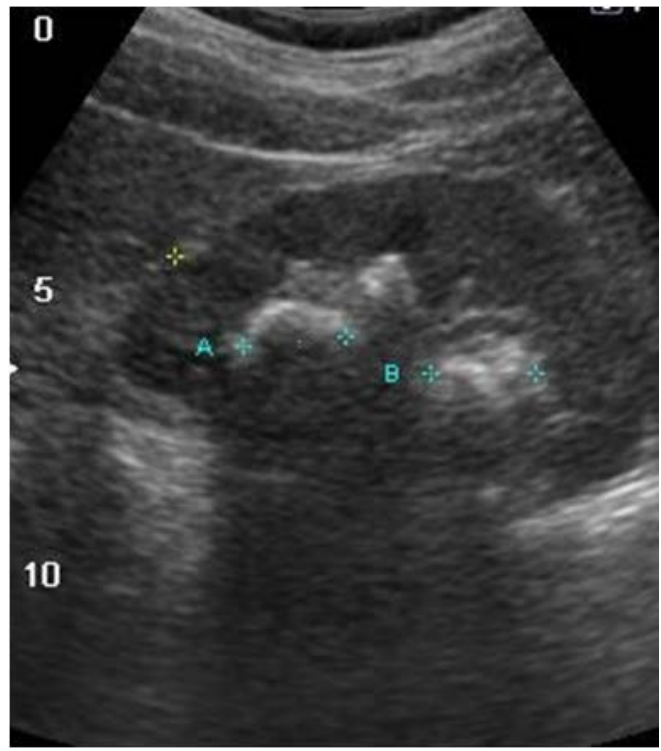

Figure 5. Multiple hyperechoic areas lying adjacent to each other which may suggest different limbs of staghorn calculus or different calculi lying adjacent to each other.

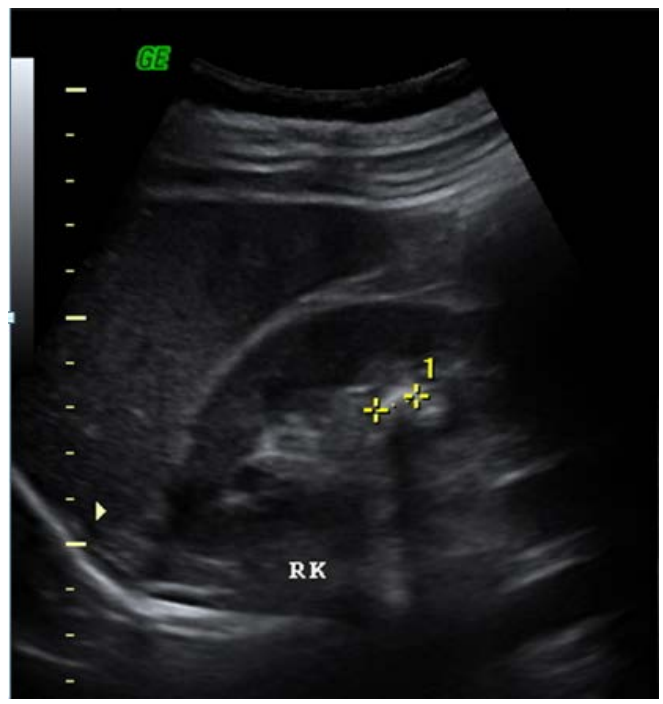

Figure 6. Renal calculus (between callipers) with shadow.

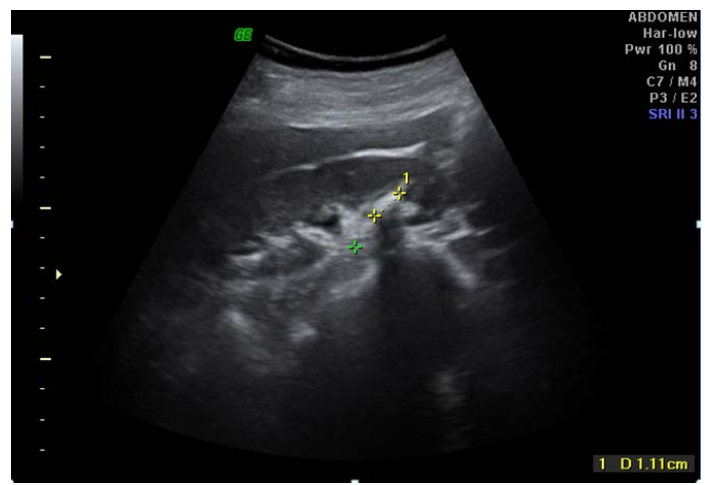

Figure 7. Renal calculus (between callipers) without any evidence of hydronephrosis. Acoustic shadowing is seen deep to the calculi. 


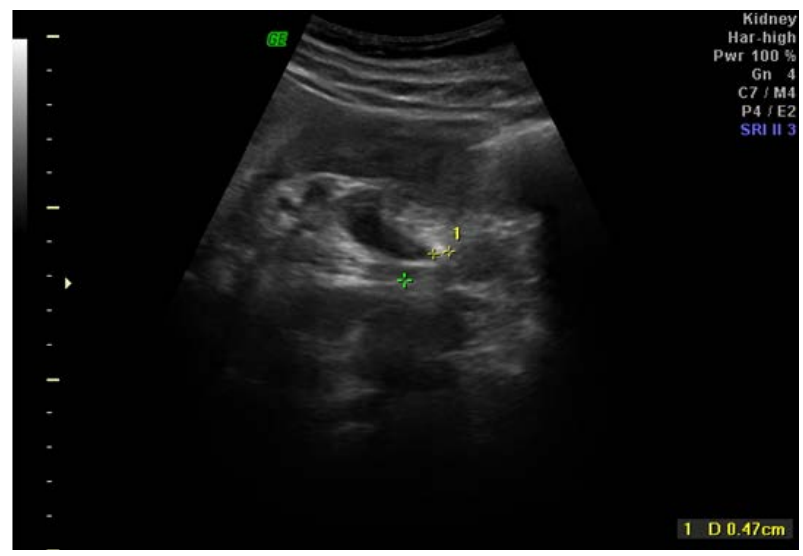

Figure 8. Ureteric calculus (between calipers) with hydronephrosis.

example uric acid stones IVP is something we used to use but involved exposure too much radiation. Ultrasound can be helpful especially in the absence of helical CT or in country and contraindication for example in pregnancy in ultrasound this is usually indicated and with the CT its best not to use contrast. [5] We don't use contrast because that could make it the stones don't show up [17]. They get lost in the contrast and the MRI is not really used to it except for in situations where there's a contraindication to CT for example pregnancy but a stone cannot be visualized on ultrasound.

THERAPY: The biggest part of treatment for nephrolithiasis is pain management and hydration these people come in a lot of pain and that's the most important thing to take care of especially as most of them are not in any kind of urgent danger. hydration also helps to dilute the urine which can in some case lead to the dissolving of stones overtime and prevention of increased stones and the most important thing is just to take care of whatever is causing the stones to begin with there are some cases where you want to get urgent urologic consult if there's evidence of urosepsis or renal failure or if the patient is just really sick then you want to get a urologic consult these patients should be staining their urine in order to help identify the stones if we don't know what kind of stone it is and help us to prevent in the future and in some case we do need to facilitate the passage of stones you can get these stone to pass in a number of different ways if they're not passing all by themselves medically you can give anti-spasmodic in order to allow the smooth muscle to relax in the ureter to make more space for the stone to pass through calcium channel blockers [20] and steroids have a similar effect there's different types of lithotripsy which break up the stones a shock wave lithotripsy or ureteroscopic lithotripsy and with ureteroscopic lithotripsy you can use either a laser or hydraulic pulse lithotripsy and in some cases rare cases then you got to go in and get this stone with surgery. Shockwave lithotripsy is the first line treatment in worldwide [21]. Shockwave Lithotripsy (SWL) is a non-surgical technique to treat kidney stones using high-energy shock waves [22]. A surgeon does the procedure in a hospital operating room or an outpatient surgery center. An SWL typically takes 60 minutes. 
sometimes surgeon insert a small plastic tube called a ureteral stent in your urinary tract just prior to SWL the stent typically remains in your body for 4 to 7 days and it said removed during a follow-up appointment after you are under anesthesia you will be positioned on an operating table with a water filled cushion once the stone is located shockwaves from outside the body are targeted at a kidney stone causing the stone to fragment [2]. Stones are broken into fragments that are generally small enough to pass in urine if large pieces remain ureteroscopy or another shockwave procedure can be performed [23].

Percutaneous nephrostomy allows placement of a small flexible rubber tube a catheter through the skin and into the kidney and this is an order to drain urine out if there is signs of obstruction this is more of a symptomatic relief in terms of removal of the kidney stone there is ureteric stent insertion [24]. The ureter and let us say the stone is lodged within the ureter well a stent a rod can be fed up through the urethra through the bladder and up the ureter to the side of obstruction and the stent can be placed the stent will allow drainage of the urine essentially bypassing the blockage. The urine can then just drain straight into the bladder. If the urinary stone is within the kidney a procedure called a percutaneous nephrolithotomy can be performed. In this procedure the aim is to remove the stone from the kidney by a small puncture wound through the skin. It is most suitable for removal of stones that are more than let's say 2 centimeters in size and which are present around the pelvic region of the kidney [25]. Another surgical procedure that can be done for urinary stones within the ureter or within the kidneys is a simple endoscopy procedure that will break down the stone within the kidney or the ureter alternative there is open surgery to remove and break the stone. Finally there is the extracorporeal shock wave lithotripsy which uses shock waves to break up stones that form in the kidney to enable easy passage of these fragments out of the body within urine.

FOLLOW UP TREATMENT: Once the stone is taken care of the main concern for the patient and for years the doctor treatment patient is to make sure that this doesn't happen another time which usually is going to be figuring out what the underlying causes is and keeping it from happening again. So, hypercalcemia you want to take care of the calcium levels hypercalcemia you can give thiazide diuretics which prevent the secretion or the secretion of calcium the low sodium diet does the same thing. If they have hyperuricosuria then allopurinol can be used, hypercystinuria then alkalinization can be used, struvite stone just preventing UTIs in the future it is going to be the most important with all of these fluids is an important factor to be taking in as much as possible.

\section{Conclusion}

Urolithiasis or nephrolithiasis is a common disease to be presented in emergency rooms and sometimes primary care offices. It is a condition associated with considerable pain but very little mortality in comparison; most of these people presented in a considerable amount of pain. The pain is along the side both in front 
and back and it can extend down into the pelvic region. The majority of them also have blood in the urine. Noncontrast computed tomography (CT) is the gold standard for the diagnosis. Shocking wave lithotripsy is the first line treatment. There are different types of stones which can be found in the kidney, so it is very important to find out what kind of stone it is.

\section{Conflicts of Interest}

The authors declare no conflicts of interest regarding the publication of this paper.

\section{References}

[1] Leveridge, M., D’Arcy, F.T., O’Kane, D., Ischia, J.J., Webb, D.R., Bolton, D.M., et al. (2016) Renal Colic: Current Protocols for Emergency Presentations. European Journal of Emergency Medicine, 23, 2-7. https://doi.org/10.1097/MEJ.0000000000000324

[2] Goldfarb, D.S., Avery, A.R., Beara-Lasic, L., Duncan, G.E. and Goldberg, J. (2019) A Twin Study of Genetic Influences on Nephrolithiasis in Women and Men. Kidney International Reports, 4, 535-540. https://doi.org/10.1016/j.ekir.2018.11.017

[3] Krajewski, W., Wojciechowska, J., Dembowski, J., Zdrojowy, R. and Szydelko, T. (2017) Hydronephrosis in the Course of Ureteropelvic Junction Obstruction: An Underestimated Problem? Current Opinions on the Pathogenesis, Diagnosis and Treatment. Advances in Clinical and Experimental Medicine, 26, 857-864. https://doi.org/10.17219/acem/59509

[4] Leung, V.Y.-F., Chu, W.C.-W., Yeung, C.-K. and Metreweli, C. (2007) Doppler Waveforms of the Ureteric Jet: An Overview and Implications for the Presence of a Functional Sphincter at the Vesicoureteric Junction. Pediatric Radiology, 37, 417-425. https://doi.org/10.1007/s00247-007-0433-1

[5] Ennis, J.L. and Asplin, J.R. (2016) The Role of the 24-h Urine Collection in the Management of Nephrolithiasis. International Journal of Surgery, 36, 633-637. https://doi.org/10.1016/j.ijsu.2016.11.020

[6] Schmidt-Bowman, M., Reinstatler, L., Raffin, E.P., Yared, J.E., Seigne, J.D. and Sverrisson, E.F. (2018) Acquired Hemophilia Presenting as Gross Hematuria Following Kidney Stone-A Case Report and Review of the Literature. International Brazilian Journal of Urology, 44, 390-392. https://doi.org/10.1590/s1677-5538.ibju.2017.0172

[7] Koh, Y., Imanaka, T., Tsujimura, G., Kinjo, T., Nomura, H., Yoshioka, I., et al. (2018) A Case Report: Lithiasis of the Left Ectopic Pelvic Kidney. Nihon Hinyokika Gakkai Zasshi, 109, 54-57. https://doi.org/10.5980/jpnjurol.109.54

[8] Siener, R. (2018) Dietary Treatment of Metabolic Acidosis in Chronic Kidney Disease. Nutrients, 10, 512. https://doi.org/10.3390/nu10040512

[9] Maalouf, N.M., Moe, O.W., Adams-Huet, B. and Sakhaee, K. (2011) Hypercalciuria Associated with High Dietary Protein Intake Is Not Due to Acid Load. The Journal of Clinical Endocrinology \& Metabolism, 96, 3733-3740.

https://doi.org/10.1210/jc.2011-1531

[10] Hiatt, R.A., Dales, L.G., Friedman, G.D. and Hunkeler, E.M. (1982) Frequency of Urolithiasis in a Prepaid Medical Care Program. American Journal of Epidemiology, 115, 255-265. https://doi.org/10.1093/oxfordjournals.aje.a113297 
[11] Curhan, G.C., Willett, W.C., Speizer, F.E., Spiegelman, D. and Stampfer, M.J. (1997) Comparison of Dietary Calcium with Supplemental Calcium and Other Nutrients as Factors Affecting the Risk for Kidney Stones in Women. Annals of Internal Medicine, 126, 497-504. https://doi.org/10.7326/0003-4819-126-7-199704010-00001

[12] Li, K., Wang, X.-F., Li, D.-Y., Chen, Y.-C., Zhao, L.-J., Liu, X.-G., et al. (2018) The Good, the Bad, and the Ugly of Calcium Supplementation: A Review of Calcium Intake on Human Health. Clinical Interventions in Aging, 13, 2443-2452. https://doi.org/10.2147/CIA.S157523

[13] Carbone, A., Al Salhi, Y., Tasca, A., Palleschi, G., Fuschi, A., De Nunzio, C., et al. (2018) Obesity and Kidney Stone Disease: A Systematic Review. Minerva Urologica e Nefrologica, 70, 393-400. https://doi.org/10.23736/S0393-2249.18.03113-2

[14] Mehta, M., Goldfarb, D.S. and Nazzal, L. (2016) The Role of the Microbiome in Kidney Stone Formation. International Journal of Surgery, 36, 607-612. https://doi.org/10.1016/j.ijsu.2016.11.024

[15] Diri, A. and Diri, B. (2018) Management of Staghorn Renal Stones. Renal Failure, 40, 357-362. https://doi.org/10.1080/0886022X.2018.1459306

[16] His, R.S., Sanford, T., Goldfarb, D.S. and Stoller, M.L. (2017) The Role of the 24-Hour Urine Collection in the Prevention of Kidney Stone Recurrence. Journal of Urology, 197, 1084-1089. https://doi.org/10.1016/j.juro.2016.10.052

[17] McCarthy, C.J., Baliyan, V., Kordbacheh, H., Sajjad, Z., Sahani, D. and Kambadakone, A. (2016) Radiology of Renal Stone Disease. International Journal of Surgery, 36, 638-646. https://doi.org/10.1016/j.ijsu.2016.10.045

[18] Hazar, I.A., Cakiroglu, B., Sinanoglu, O., Akgun, F.S., Arda, E., Yuksel, I., et al. (2018) Does Mild Hydronephrosis Induced by Full-Bladder Improve Outcomes in Patients Undergoing Shock Wave Lithotripsy for Lower Calyceal Stones? A Prospective Randomized Study. Urology Journal, 15, 92-95.

[19] Sun, W., Liu, M.-N., Yang, Z.-W., Wang, Q. and Xu, Y. (2017) Ultrasound-Guided Percutaneous Nephrolithotomy for the Treatment in Patients with Kidney Stones. Medicine (Baltimore), 96, e9232. https://doi.org/10.1097/MD.0000000000009232

[20] Zisman, A.L. (2017) Effectiveness of Treatment Modalities on Kidney Stone Recurrence. Clinical Journal of the American Society of Nephrology, 12, 1699-1708. https://doi.org/10.2215/CJN.11201016

[21] Yamashita, S., Kohjimoto, Y., Iwahashi, Y., Iguchi, T., Nishizawa, S., Kikkawa, K., et al. (2018) Noncontrast Computed Tomography Parameters for Predicting Shock Wave Lithotripsy Outcome in Upper Urinary Tract Stone Cases. BioMed Research International, 2018, Article ID: 9253952. https://doi.org/10.1155/2018/9253952

[22] Chaussy, C.G. and Tiselius, H.-G. (2018) How Can and Should We Optimize Extracorporeal Shockwave Lithotripsy? Urolithiasis, 46, 3-17.

https://doi.org/10.1007/s00240-017-1020-z

[23] Li, T., Gao, L., Chen, P., Bu, S., Cao, D., Yang, L., et al. (2016) Supine versus Prone Position during Extracorporeal Shockwave Lithotripsy for Treating Distal Ureteral Calculi: A Systematic Review and Meta-Analysis. Urologia Internationalis, 97, 1-7. https://doi.org/10.1159/000439140

[24] Xu, C., Feng, S., Lin, C. and Zheng, Y. (2018) Reducing Postoperative Morbidity of Mini-Invasive Percutaneous Nephrolithotomy: Would It Help If Blood Vessels Are Left Unharmed during Puncture? A Consort-Prospective Randomized Trial. Medicine (Baltimore), 97, e13314. https://doi.org/10.1097/MD.0000000000013314

[25] Iordache, A., Baston, C., Guler-Margaritis, S.-S., Angelescu, E., Cerempei, V., Olivi- 
er, T., et al. (2018) Ultrasound for Kidney Access in Percutaneous Nephrolithotomy: A Contemporary Review. Medical Ultrasonography, 20, 508-514.

https://doi.org/10.11152/mu-1618 